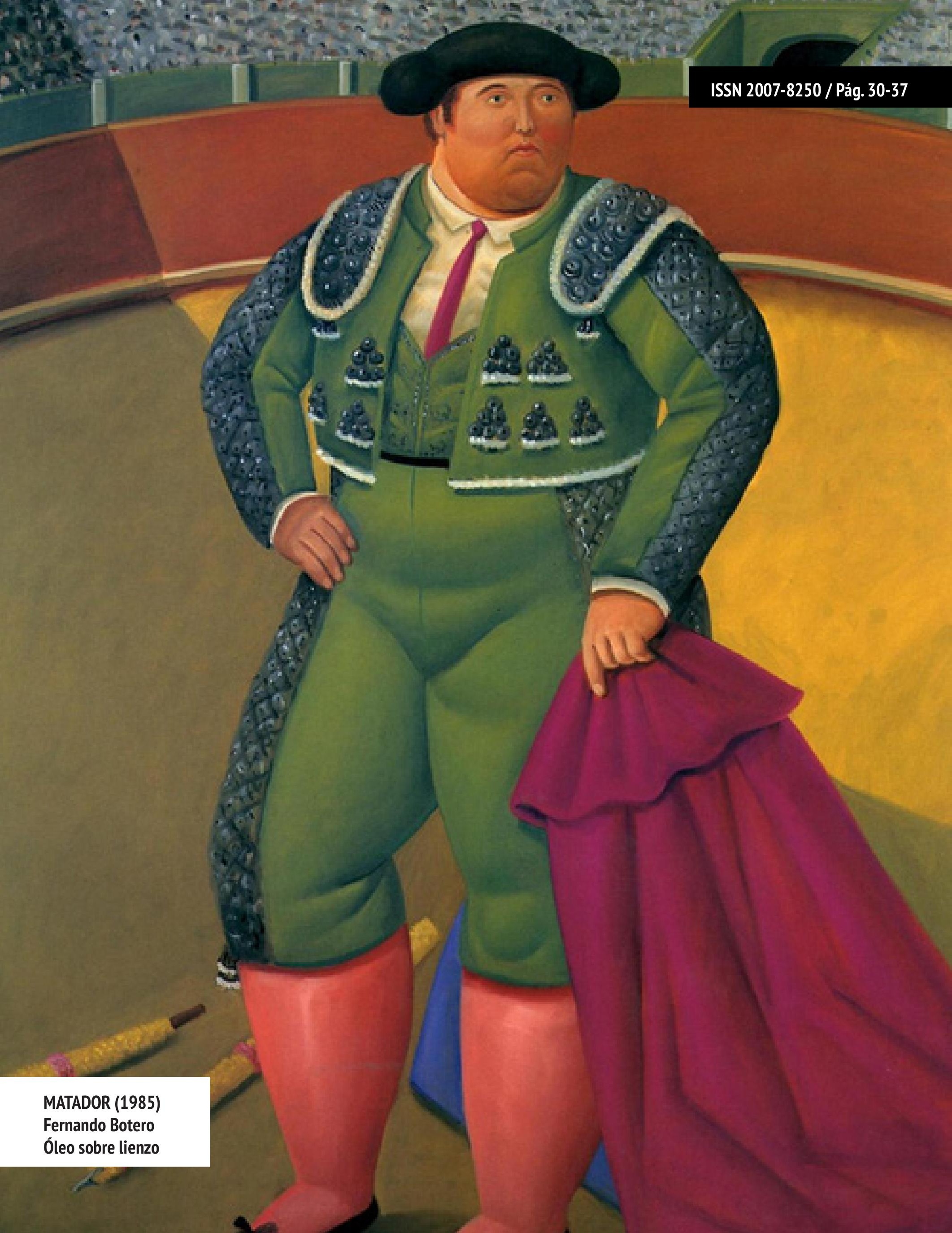




\section{HABILIDADES METACOGNITIVAS EN ESTUDIANTES DE BACHILLERATO}

METACOGNITIVE SKILLS IN HIGH SCHOOL STUDENTS

PEDRO ALONSO SERNA SÁNCHEZ

\section{RESUMEN}

El presente trabajo de investigación de carácter exploratorio, analizó el nivel de desarrollo de habilidades meta cognitivas en estudiantes de Bachillerato. Se utilizó como instrumento de recopilación de datos, el Inventario de Habilidades Metacognitivas (MAI), el cual se aplicó a estudiantes de cuatro grupos: tres de tercer semestre y uno de quinto semestre. Dos de los grupos de tercer semestre pertenecen al programa de Bachillerato General (BG) y el restante al programa de Bachillerato Bilingüe Progresivo (BBP). El grupo de quinto semestre cursaba el Programa de Bachillerato Técnico en Diseño Gráfico (BT). Se trabajó con una población escolar total de 131 estudiantes. El instrumento constó de 52 ítems, de los cuales 17 correspondieron a la dimensión Conocimiento de la cognición y 35 a la dimensión Regulación de la cognición. Cada dimensión se desglosó en subprocesos específicos. Los resultados muestran que, en general, la dimensión Conocimiento de la cognición presentó puntajes más altos que la relacionada con la Regulación de la cognición. De esta última, los procesos relacionados con la evaluación, la planificación y el monitoreo de la comprensión, presentaron los puntajes más bajos. Al realizar pruebas comparativas entre hombres y mujeres, no se encontraron diferencias estadísticamente significativas. De la misma forma, de los grupos considerados en el estudio, el correspondiente al Bachillerato Técnico de quinto semestre presentó puntajes superiores sólo en algunos de los subconjuntos analizados. En el resto de las comparaciones entre grupos no se encontraron diferencias estadísticamente significativas.

PALABRAS CLAVE: Regulación - Metacognición - Habilidades Metacognitivas - Cognición - Aprendizaje.
ABSTRACT

The present exploratory research work analyzed the level of development of meta-cognitive skills in high school students. The Metacognitive Skills Inventory was used as a data collection instrument, which was applied to students from four groups: three from the third semester and one from the fifth semester. Two of the third semester groups belong to the General Baccalaureate program and the rest to the Progressive Bilingual Baccalaureate program. The fifth semester group was studying the Technical Baccalaureate Program in Graphic Design. We worked with a total school population of 131 students. The instrument consisted of 52 items, of which 17 corresponded to the Knowledge of cognition dimension and 35 to the Regulation of cognition dimension. Each dimension was broken down into specific threads. The results show that, in general, the Knowledge of cognition dimension presented higher scores than the one related to the Regulation of cognition. The processes related to the evaluation, planning and monitoring of comprehension, presented the lowest scores. When performing comparative tests between men and women, no statistically significant differences were found. In the same way, of the groups considered in the study, the one corresponding to the Technical Baccalaureate of the fifth semester presented higher scores only in some of the subsets analyzed. In the rest of the comparisons between groups, no statistically significant differences were found.

KEYWORDS: Regulation - Metacognition - Skills - Metacognitive - Cognition - Learning. 
L

a metacognición es un concepto que muchos consideran difuso y que tiene significados muy diversos, por lo que en los últimos años se han realizado numerosos trabajos de investigación para tratar de conocer más su estructura (González, 1993; Akturk and Sahin, 2011). Incluso se ha señalado la dificultad de medirla de tal forma que se logre una conexión simultánea entre todos los procesos que la constituyen (Schraw, 2009). En general, la metacognición se refiere al conocimiento que una persona tiene sobre los propios procesos y productos cognitivos, es decir, las propiedades de la información o los datos relevantes para su aprendizaje; se refiere al control, orquestación y regulación subsiguiente de estos procesos (Flavell, 1976; Livingston, 2003). Determinados recursos y actividades tienen carácter metacognitivo en la medida en que inciden en el conocimiento 0 control del propio conocimiento 0 procesos cognitivos, en la autorregulación cognitiva y en las ideas adecuadas sobre la estructura, producción y organización del conocimiento (Campanario, 2000). Así, los altos niveles de desarrollo de las habilidades metacognitivas de los estudiantes están asociadas a elevados niveles de logro académico, consecuencia de un mayor desarrollo de un aprendizaje autónomo, autorregulado y sostenido.

\section{PLANTEAMIENTO DEL PROBLEMA}

El desarrollo de habilidades metacognitivas en los estudiantes promueve en ellos el pensamiento crítico y el análisis reflexivo, lo cual posibilita el logro de un aprendizaje autónomo y autorregulado, en congruencia con el nuevo perfil del egresado del Bachillerato. En este contexto, el presente trabajo de investigación realiza el si-

La metacognición se refiere al conocimiento que una persona tiene sobre los propios procesos y productos cognitivos, es decir, las propiedades de la información o los datos relevantes para su aprendizaje; se refiere al control, orquestación y regulación subsiguiente de estos procesos

guiente planteamiento del problema: ¿Cuál es el nivel de desarrollo de habilidades metacognitivas de los estudiantes de Bachillerato?

\section{OBJETIVO GENERAL DE LA INVESTIGACIÓN}

Explorar el nivel de desarrollo de habilidades metacognitivas que presentan los estudiantes de Bachillerato.

\section{OBJETIVOS ESPECÍFICOS DE LA INVESTIGACIÓN}

1. Determinar los procesos metacognitivos en los que los estudiantes de bachillerato presentan mayores fortalezas y deficiencias.

2. Detectar posibles diferencias en el desarrollo de habilidades metacognitivas de los estudiantes relacionados con el factor género.

3. Explorar el nivel de desarrollo de las habilidades metacognitivas en los estudiantes en función del programa educativo cursado en el bachillerato.

\section{HIPÓTESIS}

Existen algunas diferencias significativas en los niveles de desarrollo de habilidades metacognitivas de los estudiantes de bachillerato, diferencias en función de diversos factores como el programa educativo y el género.

\section{JUSTIFICACIÓN DE LA INVESTIGACIÓN}

Al reflexionar sobre los procesos de enseñanza y aprendizaje implementados en las instituciones educativas basados en modelos que deban dar respuesta a las necesidades de los nuevos contextos y entornos globales, se deduce que para ser congruente con esta nueva realidad se debe fomentar un aprendizaje autónomo, autorregulado y continuo, que permita al estudiante orientarse en su propio proceso de formación. En este contexto, se ha vuelto una necesidad imperiosa la de aprender a aprender, permitiendo que cada individuo organice y administre su propio proceso de aprendizaje. Por ello, cualquier sistema educativo debe fomentar la formación de estudiantes dotados de un pensamiento flexible, con capacidad de cambio continuo y manejo de las estrategias adecuadas para la regulación y ordenación del propio aprendizaje. En este marco de referencia, la utilización de estrategias cognitivas y metacognitivas permiten al estudiante conocer cómo adquiere el conocimiento a partir de la 
conciencia de sus propias fortalezas y debilidades y actuar de manera consciente y regulada en los procesos de planeación, monitoreo y evaluación de sus aprendizajes, sentando las bases para el logro de un aprendizaje autónomo y autorregulado.

\section{MARCO TEÓRICO}

La metacognición está relacionada con los procesos reflexivos de las personas sobre su propio conocimiento y lo que saben acerca de su propia actividad cognitiva. Así, los dos elementos que conforman la metacognición son la conciencia del propio conocimiento y la regulación que sobre éstos se ejerce (Huertas Bustos, Vesga Bravo y Galindo León, 2014). El conocimiento de la cognición se refiere al conocimiento que tienen las personas sobre la cognición en general y tiene como subprocesos el conocimiento declarativo (saber sobre), el conocimiento procedimental (saber cómo) y el conocimiento condicional (saber por qué y cuándo). Por su parte, la regulación de la cognición está relacionada con las actividades metacognitivas que ayudan a controlar el aprendizaje y está conformada por los subprocesos: Planeación, Monitoreo y Evaluación (Schraw and Moshman, 1995), así como por la Organización y la Depuración (Schraw and Denninson, 1994).

Diversos trabajos de investigación han demostrado la importancia del desarrollo de habilidades metacognitivas en los estudiantes, ya que está directamente relacionado con el logro académico, el aprendizaje autorregulado y el desarrollo del pensamiento crítico (Klimenko y Alvares, 2009; Magno, 2010; Doganay y Demir, 2011; Bogdanovic et al. 2015). Para examinar la conciencia metacognitiva de los estudiantes, se ha utilizado frecuentemente el Inventario de Habilidades Metacognitivas (MAI), creado y validado por Schraw y Denninson (1994). Este instrumento ha sido utilizado ampliamente en diversas investigaciones, especialmente para demostrar la relación de la metacognición con el logro del aprendizaje (Young y Fry, 2008; Gula y Shehzadb, 2012; Narang y Saini, 2013).

\section{SELECCIÓN DE LA MUESTRA}

El presente trabajo de investigación se llevó a cabo en la Escuela Preparatoria 24 Dr. Alfredo Piñeyro López de la UANL ubicada en el municipio de Anáhuac N.L., durante el semestre Agosto - Diciembre 2018. Se trabajó con 4 grupos de estudiantes: 2 grupos del programa de Bachillerato General (301BG y 302BG, con 34 y 33 estudiantes respectivamente), un grupo

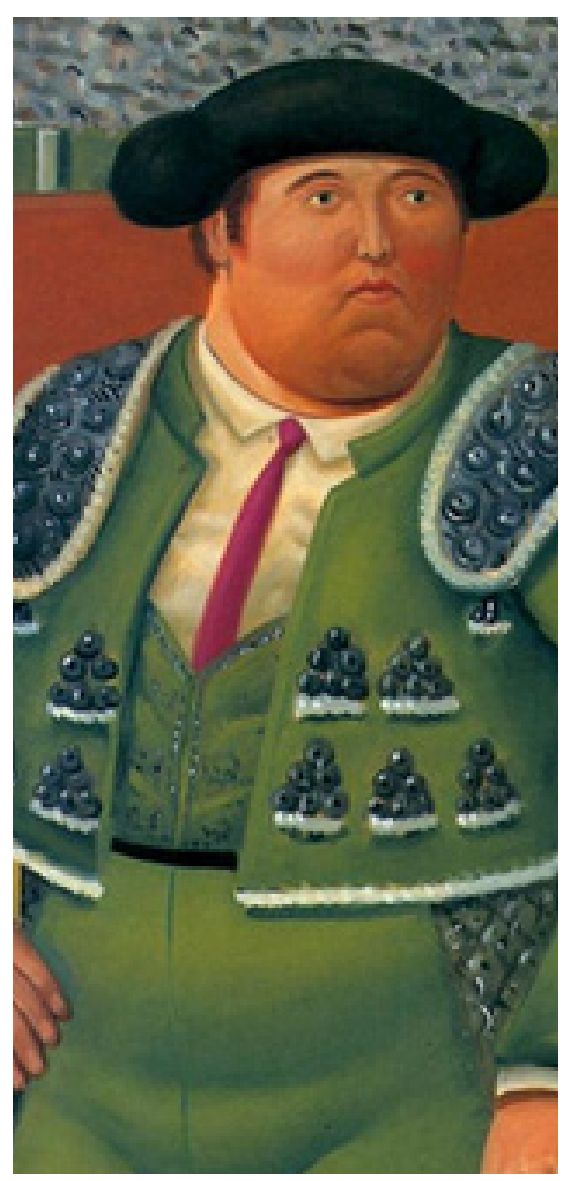

del Bachillerato Bilingüe Progresivo (303BBP con 36 estudiantes), los 3 de 3er semestre, así como un grupo del Bachillerato Técnico en Diseño Gráfico (503BT con 28 estudiantes) de 5to semestre, dando una población escolar total de 131 estudiantes.

\section{INSTRUMENTO DE INVESTIGACIÓN}

El instrumento utilizado fue el Inventario de Habilidades Metacognitivas (MAI) de Schraw y Dennison (1994), versión en español adaptada y validada por Huertas Bustos, Vesga Bravo y Galindo León (2014). Consta de 52 ítems, de los cuales 17 correspondieron a la dimensión Conocimiento de la Cognición y 35 a la dimensión Regulación de la Cognición. La primera dimensión se desglosó en los subprocesos: Conocimiento Declarativo, Conocimiento Procedimental y Conocimiento Condicional. En relación a la segunda dimensión, se dividió en los subprocesos: Planificación, Estrategias de Manejo de la Información, Monitoreo de la Comprensión, Estrategias de Depuración y Evaluación. Para cada ítem se utilizó una escala de 0 a 10 , en donde el 0 corresponde a totalmente en desacuerdo y el 10 a totalmente de acuerdo. Se realizó una prueba piloto para determinar la confiabilidad del instrumento en un grupo que no formó parte de la investigación. Se obtuvo un coeficiente alfa de Cronbach de 0.919 para todo el instrumento en la prueba piloto y de 0.936 durante toda la investigación.

\section{PROCEDIMIENTO}

El instrumento se aplicó una sola vez a los estudiantes de los 4 grupos. Los datos recabados se analizaron utilizando el paquete estadístico SPSS versión 19. Se obtuvieron los puntajes totales expresados en porcentajes 
para cada una de las 2 dimensiones de la Metacognición y para cada uno de los subprocesos. Se procesaron pruebas de normalidad a todos los conjuntos de datos analizados a través de las pruebas de Shapiro y Wilk y Kolmogorov Smirnov. Se llevaron a cabo análisis descriptivos para cada una de las dimensiones y subprocesos de la Metacognición, así como análisis comparativos utilizando la prueba $t$ para muestras independientes y/o la prueba U de Mann Whitney, con el fin de contrastar las variables analizadas entre los grupos considerados pertenecientes a cada programa educativo, así como para establecer diferencias tomando como factor el género. Para las pruebas de hipótesis se utilizó un nivel de significancia de $p \leqslant 0.05$.

\section{RESULTADOS}

Considerando los 4 grupos en su conjunto, la dimensión Regulación de la Cognición presentó la media más baja $(M=67.692)$ en relación a la dimen- sión Conocimiento de la Cognición ( $M$ $=77.925$ ). Ambas dimensiones mostraron asimetría negativa con valores entre -0.50 y 0 . En relación al análisis descriptivo de los subprocesos en ambas dimensiones, la Tabla 1 muestra los puntajes totales expresados en porcentajes para cada uno de ellos. El subproceso Evaluación presentó la media más baja ( $M=60.445)$, seguido de los subprocesos Planificación ( $M=$ 62.868) y Monitoreo de la Comprensión ( $M=69.367)$, mientras que la variable Conocimiento Declarativo presentó la media más alta $(M=80.935)$. La totalidad de los subprocesos presentaron asimetría negativa con tendencia a valores altos de la escala.

Al realizar pruebas comparativas entre los diferentes grupos en relación a los 8 subprocesos pertenecientes a ambas dimensiones, se encontró que el Grupo 503BT del Bachillerato Técnico presentó medias estadísticamente superiores que las presentadas por los
Grupos 301BG (M 503BT = 79.017 vs $M 301 B G=71.838)$ y $303 \mathrm{BBP}$ del $\mathrm{Ba}-$ chillerato Bilingüe Progresivo (M 503 $\mathrm{BT}=79.017$ vs $\mathrm{M} 303 \mathrm{BBP}=69.861$ ) en la variable Conocimiento Procedimental.

De la misma forma, de nuevo el Grupo 503BT presentó una media estadísticamente superior que el Grupo 301BG en la variable Estrategias de manejo de la Información (M 503BT = 73.785 vs M 301BG = 67.029). Adicionalmente, el Grupo 302BG presentó una media estadísticamente superior a la obtenida por el Grupo 301BG en la variable Estrategias de Depuración (M 302BG $=80.545$ vs $M 301 B G=73.294)$. En el resto de las comparaciones entre grupos no se encontraron diferencias significativas ( $p \leqslant 0.05)$.

Por otro lado, no se encontraron diferencias estadísticamente significativas entre los hombres y las mujeres, independientemente del grupo al que

Tabla 1. Estadística descriptiva para los subprocesos de las dos dimensiones de la metacognición, considerando los 4 grupos de estudio en su conjunto.

\begin{tabular}{|c|c|c|c|c|c|c|c|c|c|}
\hline Subproceso & $\mathbf{N}$ & Mín & Máx & $M$ & DT & As & K & KS & $P$ \\
\hline $\begin{array}{l}\text { A. Conocimiento } \\
\text { declarativo }\end{array}$ & $|3|$ & 51.25 & 98.75 & 80.935 & 9.203 & -.415 & -.021 & .079 & .046 \\
\hline $\begin{array}{l}\text { A. Conocimiento } \\
\text { procedimental }\end{array}$ & $|3|$ & 25.00 & 100.00 & 73.263 & 13.448 & -.707 & 1.036 & .086 & .020 \\
\hline $\begin{array}{l}\text { A. Conocimiento } \\
\text { condicional }\end{array}$ & $|3|$ & 20.00 & 100.00 & 76.839 & 13.596 & $-|.04|$ & 2.162 & .124 & .000 \\
\hline B. Planificación & $|3|$ & 22.86 & 95.71 & 62.868 & |7.04| & -.343 & -.584 & .095 & .005 \\
\hline $\begin{array}{l}\text { B. Est. de Manejo } \\
\text { de Información }\end{array}$ & $|3|$ & 32.00 & 98.00 & 70.885 & 12.511 & -.379 & -.163 & .066 & $.200^{*}$ \\
\hline $\begin{array}{l}\text { B. Monitoreo } \\
\text { de la Comprensión }\end{array}$ & $|3|$ & 28.57 & 98.57 & 69.367 & 13.938 & -.503 & $-.07 \mid$ & .093 & .007 \\
\hline $\begin{array}{l}\text { B. Estrategias } \\
\text { de depuración }\end{array}$ & $|3|$ & 32.00 & 100.00 & 77.450 & 13.936 & -.996 & 1.216 & .130 & .000 \\
\hline B. Evaluación & $|3|$ & .00 & 91.67 & 60.445 & I7.92I & $-.60 \mathrm{I}$ & .054 & .096 & .004 \\
\hline
\end{tabular}

Nota: $\mathrm{M}=$ media aritmética, $\mathrm{DT}$ = desviación estándar, $\mathrm{As}=$ asimetría, $\mathrm{K}=$ curtosis, $\mathrm{KS}=$ Kolmogorov Smirnov, $\mathrm{p}=$ nivel de probabilidad. Fuente: Inventario sobre Habilidades Metacognitivas MAl (adaptación de Huertas Bustos, Vesga Bravo y Galindo León, 2014). A = Dimensión: Conocimiento de la Cognición y B = Dimensión: Regulación de la Cognición. 
Tabla 2. Prueba t para muestras independientes para los puntajes totales de los subprocesos de las 2 dimensiones de la metacognición, considerando los 4 grupos de estudio.

\begin{tabular}{|c|c|c|c|c|c|c|c|c|c|}
\hline \multirow{2}{*}{\multicolumn{2}{|c|}{$\begin{array}{l}\text { Subprocesos } \\
\text { de la } \\
\text { Metacognición }\end{array}$}} & \multirow[t]{2}{*}{ Grupo } & \multirow[t]{2}{*}{$\mathbf{N}$} & \multirow[t]{2}{*}{$M$} & \multirow[t]{2}{*}{$\mathrm{t}$} & \multirow{2}{*}{$\mathrm{P}$} & \multirow[t]{2}{*}{ DT } & \multicolumn{2}{|c|}{$\begin{array}{c}\text { Homogeneidad } \\
\text { de Varianzas }\end{array}$} \\
\hline & & & & & & & & Levene & $P$ \\
\hline \multirow{4}{*}{ A. } & \multirow{4}{*}{$\begin{array}{l}\text { Conocimiento } \\
\text { procedimental }\end{array}$} & $30 I B G$ & 34 & 71.838 & \multirow[t]{2}{*}{-2.589} & \multirow[t]{2}{*}{.012} & 9.698 & \multirow[t]{2}{*}{.737} & \multirow[t]{2}{*}{.394} \\
\hline & & 503BT & 28 & 79.017 & & & 12.139 & & \\
\hline & & 303BBP & 36 & $69.86 I$ & \multirow[t]{2}{*}{-2.684} & \multirow[t]{2}{*}{.009} & 14.527 & \multirow[t]{2}{*}{.549} & \multirow[t]{2}{*}{.461} \\
\hline & & 503BT & 28 & 79.017 & & & 12.139 & & \\
\hline \multirow[t]{2}{*}{ B. } & \multirow{2}{*}{$\begin{array}{l}\text { Est. de manejo } \\
\text { de la información }\end{array}$} & 30 IBG & 34 & 67.029 & \multirow[t]{2}{*}{-2.188} & \multirow[t]{2}{*}{.033} & 13.111 & \multirow[t]{2}{*}{1.268} & \multirow[t]{2}{*}{.265} \\
\hline & & 503BT & 28 & 73.785 & & & 10.733 & & \\
\hline \multirow[t]{2}{*}{ B. } & \multirow{2}{*}{$\begin{array}{l}\text { Estrategias } \\
\text { de depuración }\end{array}$} & 30 IBG & 34 & 73.294 & \multirow[t]{2}{*}{-2.314} & \multirow[t]{2}{*}{.024} & 13.099 & \multirow[t]{2}{*}{.309} & \multirow[t]{2}{*}{.580} \\
\hline & & 302BG & 33 & 80.545 & & & 12.532 & & \\
\hline
\end{tabular}

Nota: $\mathrm{N}$ = no. de sujetos, $\mathrm{M}$ = media aritmética, $\mathrm{DT}$ = desviación estándar, $\mathrm{t}$ = estadístico de prueba, $\mathrm{p}$ = nivel de probabilidad. Fuente: Inventario sobre Habilidades Metacognitivas MAI (adaptación de Huertas Bustos, Vesga Bravo y Galindo León, M, 2014). A = Dimensión: Conocimiento de la Cognición y B = Dimensión: Regulación de la Cognición.

pertenecían, en relación a la dimensión: Conocimiento de la Cognición ( $M$ Hombres $=77.323$ vs $\mathrm{M}$ Mujeres $=78.595 ; \mathrm{t}=-0.728 ; \mathrm{p}=0.468) ;$ así como tampoco en la dimensión Regulación de la Cognición (M Hombres = 66.517 vs $\mathrm{M}$ Mujeres = 69.000; $\mathrm{t}$ = $-1.116 ; p=0.266)$. De manera similar, al realizar pruebas comparativas entre hombres y mujeres en general, en relación a los subprocesos de ambas dimensiones, tampoco se encontraron diferencias estadísticamente significativas $(p \leqslant 0.05)$ entre ambos sexos.

\section{CONCLUSIÓN}

Los estudiantes mostraron mayores niveles de desarrollo en habilidades metacognitivas relacionadas con la dimensión Conocimiento de la Cognición; es decir, en conocimientos declarativos, procedimentales y condicionales. En contraposición, los procesos relacionados con la evaluación, la planificación y el monitoreo de la comprensión, pertenecientes a la dimensión Regulación de la Cognición, presentaron las medias más bajas.
Por lo tanto, los estudiantes tienen dificultades para fijarse metas y establecer tiempos para su cumplimiento, seleccionar estrategias apropiadas y recursos (Planeación), no tienen conciencia de la comprensión y ejecución de tareas mientras se están desarrollando (Monitoreo) y muestran poca capacidad para hacer una valoración o juicio de los aprendizajes logrados y la pertinencia de las estrategias implementadas (Evaluación).

No se encontraron diferencias estadísticamente significativas entre hombres y mujeres. Al realizar comparaciones entre grupos, el perteneciente al Bachillerato Técnico presentó puntajes superiores a los otros grupos, pero sólo en las variables relacionadas con el conocimiento procedimental y con las estrategias de manejo de la información.

\section{PROPUESTAS}

A partir de los resultados de esta investigación y en relación directa con los procesos metacognitivos, se plan- tea la implementación de estrategias didácticas y actividades de aprendizaje en el aula, que promuevan en los estudiantes:

1. El establecimiento de metas a corto, mediano y largo plazo definiendo tiempos para su cumplimiento, así como el saber seleccionar estrategias apropiadas de trabajo y los recursos necesarios para su implementación.

2. La generación de un proceso de automonitoreo que les permita tener conciencia de la comprensión y ejecución de las tareas, trabajos académicos y proyectos, mientras los están realizando.

3. El desarrollo de la capacidad para hacer una valoración o juicio realista de los aprendizajes logrados y la pertinencia de las estrategias implementadas para ello.

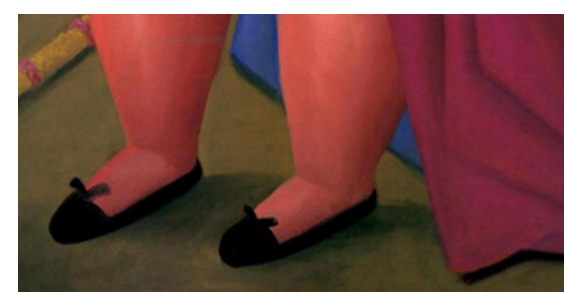




\section{REFERENCIAS}

Akturk, A.0. and Sahin, I. (2011). Literature Review on Metacognition and its Measurement. Procedia Social and Behavioral Sciences 15 (2011) 3731-3736.

Bogdanovic, I., Obadovic, E.Z., Cvjeticanin, S., Segedinac, M. and Budic, S. (2015). Students Metacognitive Awareness and Physics Learning Efficiency and Correlation between Them. European J. of Physics Education. Volume 6 Issue 2: 2015.

Campanario, J.M. (2000). El Desarrollo de la Metacognición en el Aprendizaje de las Ciencias: Estrategias para el Profesor y Actividades orientadas al Alumno. Enseñanza de las Ciencias, 18 (3), 369-380.

Doganay, A. \& Demir, 0. (2011). 'Comparison of the Level of Using Metacognitive Strategies during Study between High Achieving and Low Achieving Prospective Teachers'. Educationat Sciences: Theory \& Practice [11, 4, 2036-2043].

Flavell, J.H. (1976). Metacognitive aspects of problem solving, en Resnick, L.B. (Ed.). The nature of intelligence. Hillsdale, New Jersey: Lawrence Erlbaum.

González, F.E. (1993). Acerca de la Metacognición. Universidad Pedagógica Experimental Libertador. Paradigma. Vol. XIV al XVII. 1993-1996.

Huertas Bustos, A.P., Vesga Bravo, G.J.y Galindo León, M. (2014). Validación del Instrumento "Inventario de Habilidades Metacognitivas (MAI)" con Estudiantes Colombianos. Praxis \& Saber. Vol. 5, Num. 10. Julio-Diciembre 2014. Pág. 55-74.

Klimenko, 0. \& Álvarez, J. (2009). Aprender cómo aprendo: la enseñanza de estrategias metacognitivas'. Educación y Educadores [12, 2, 11-28].

Livingston, J.A. (2003). Metacognition: An Overview. Report. Document Resume. University of Buffalo and State University of New York.

Magno, C. (2010). 'The role of metacognitive skills in developing critical thinking'. Metacognition Learning [5, 137-156].

Schraw, G. \& Dennison, R. (1994). 'Assessing metacognitive awareness'. Contemporary Educational Psychology [19, 460-475].

Schraw, G. \& Moshman, D. (1995). "Metacognitive Theories". Educational Psychology [7, 351-371].

Young, A. \& Fry, J. (2008). 'Metacognitive awareness and academic achievement in college students'. Journal of the Scholarship of Teaching and Learning [8, 2, 1-10].

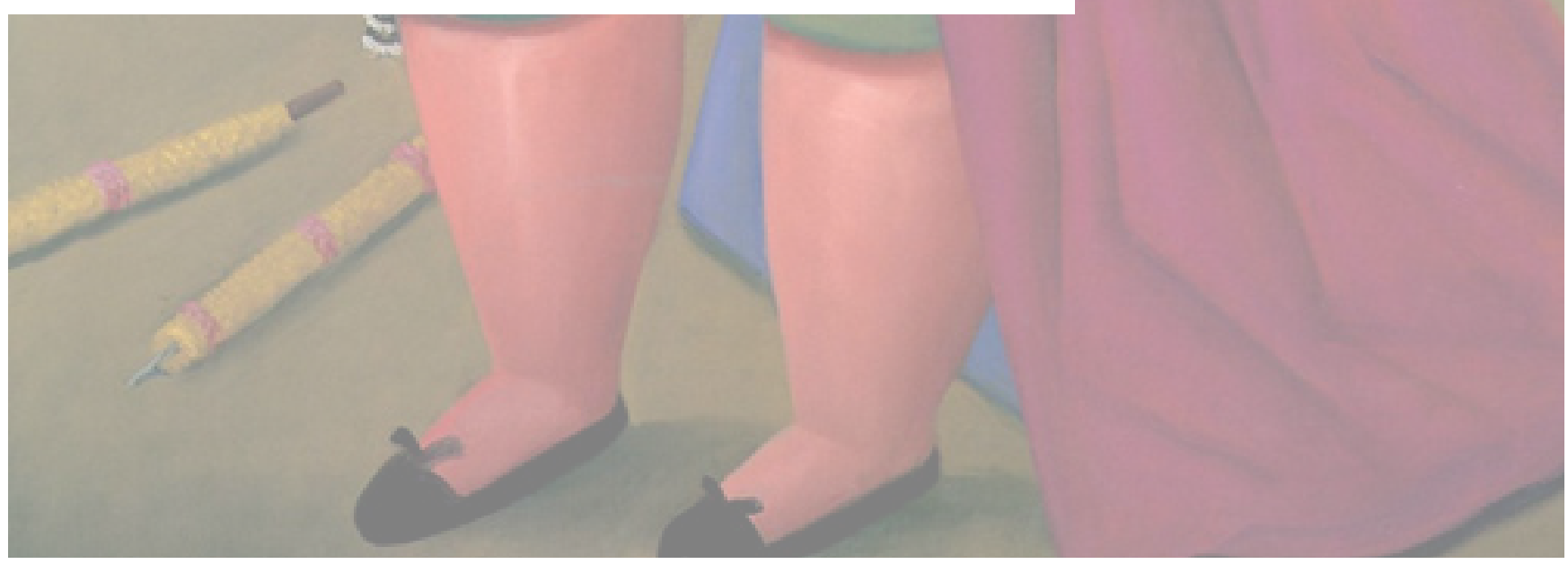




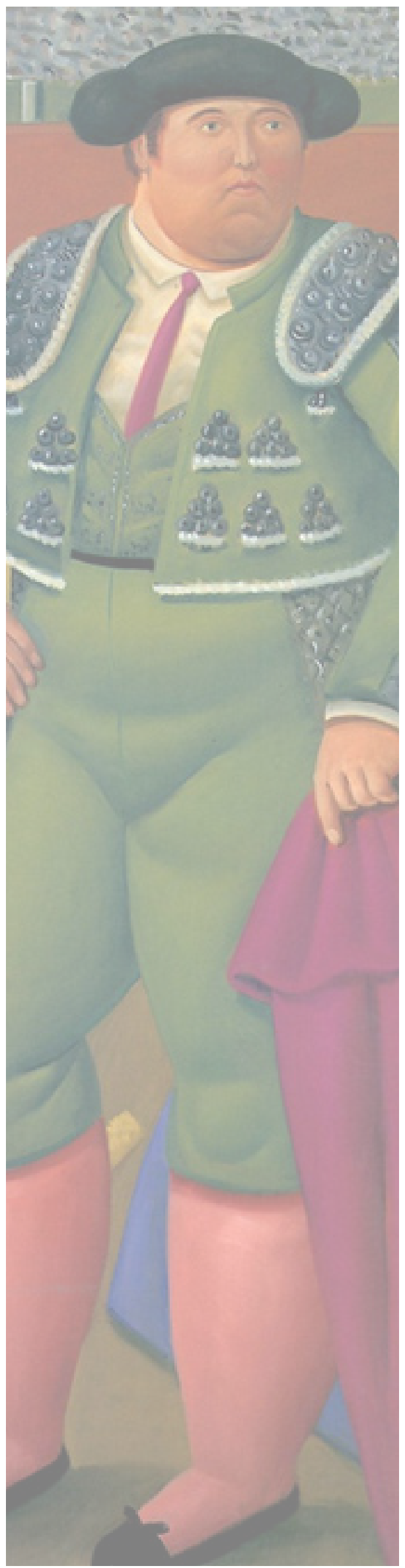

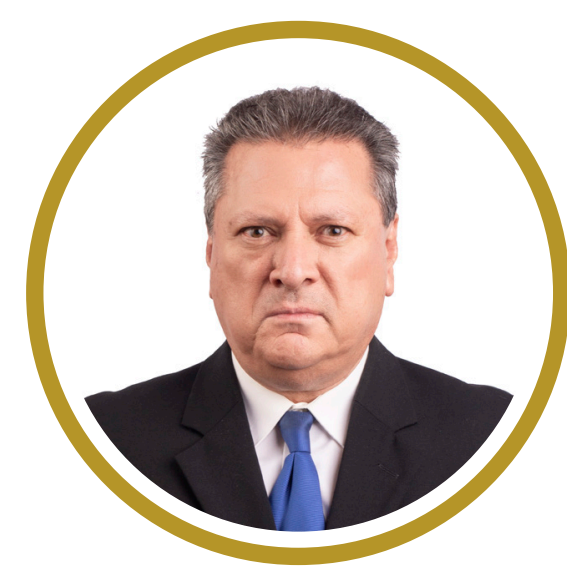

Pedro Alonso

Serna Sánchez

Ingeniero Agrónomo Zootecnista egresado de la Facultad de Agronomía de la UANL (1986); Maestría en Ciencias en Producción Animal por la Facultad Superior de Zootecnia de la Universidad Autónoma de Chihuahua (1993); Doctor en Ciencias de la Educación por la Facultad de Ciencia, Educación y Humanidades de la Universidad Autónoma de Coahuila (2017). Diplomado y Certificación en Competencias Docentes (SNB), Maestro de Tiempo Completo (desde 1988), Secretario Académico y Administrativo (19901997) y Director de la Escuela Preparatoria 24 Dr. Alfredo Piñeyro López, UANL (1997-2003). Es miembro de la Sociedad Mexicana de Física (SMF) y de la American Association of Physics Teachers (AAPT). Recientemente, el $\mathrm{H}$. Consejo Universitario le otorgó el Reconocimiento como Profesor Emérito de la UANL, en Sesión Solemne realizada el 17 de septiembre de 2020.

\section{Correo Electrónico:}

pa.sernas@hotmail.com

Fecha de Recepción: 15/03/20

Fecha de Aprobación: 15/08/20 